\title{
Local Cerebral Blood Flow during Biofeedback Training
}

Yasuyuki Matsuura ${ }^{1,2}$, Toru Tanimura ${ }^{3}$, Daisuke lida ${ }^{3}$ and Hiroki Takada ${ }^{3^{*}}$

${ }^{1}$ Department of Cross Cultural Studies, Gifu City Women's College, Gifu, Japan

${ }^{2}$ Faculty of Humanities and Social Sciences, Prince of Songkla University, Pattani, Thailand

${ }^{3}$ Department of Human and Artificial Intelligent Systems, Graduate School of Engineering, University of Fukui, Fukui, Japan

"Corresponding author: Hiroki Takada, Department of Human and Artificial Intelligent Systems, Graduate School of Engineering, University of Fukui, Fukui, Japan, Tel: +81-776-27-8795, Fax: +81-776-27-8420; E-mail: takada@u-fukui.ac.jp

Received date: April 25, 2018; Accepted date: June 15, 2018; Published date: June 29, 2018

Copyright: ( 2018 Matsuura Y, et al. This is an open-access article distributed under the terms of the Creative Commons Attribution License, which permits unrestricted use, distribution and reproduction in any medium, provided the original author and source are credited.

\begin{abstract}
Biofeedback (BF) is a means for gaining control of bodily processes to increase relaxation, relieve pain and improve health, among other benefits. A BF instrument has three tasks. First is to monitor (in some way) a physiological process of interest. Second is to measure (quantify) what is monitored. Third is to present what is monitored or measured as meaningful information. Electromyography (EMG) and BF are often used simultaneously to handle movement during a procedure. BF is a means for gaining control of bodily processes to increase relaxation, relieve pain and improve health, among other benefits. And BF training (BFT) is not only a technique used for enhancing health, but is predicted to affect the development and maintenance of brain function. One technique for non-invasive measurement of brain activity that has been developed in recent years is brain functional imaging using near-infrared spectroscopy (NIRS). Compared to other techniques of measurement of brain activity, NIRS is a low-restraint and non-invasive measuring technique. In addition, it enables a user to take electrophysiological measurements during activity, such as training or other movements. The connection between BFT and brain function surely will garner further attention in future, as current studies state that BFT involves not only voluntary movement of the extremities and joints, but also receives input from high-level integrative functions of the brain. And furthermore, BFT, which has garnered attention in recent years, has not only revealed the importance of mental training when performing an action, but also monitors subjects' muscle performance and encourages learning of psychophysiological control. Here, we investigated the connection between BFT and local cerebral blood flow, by performing cranial NIRS with simultaneous electromyogram (EMG), recording from the rectus femoris muscle. Our results suggest that kicking action alters brain function, particularly in a manner that is predicted to activate working memory.
\end{abstract}

Keywords: Biofeedback training; Kicking action; Electromyography; Cerebral blood flow; Near-infrared spectroscopy; Mental training; Working memory

\section{Abbreviations:}

BF: Biofeedback; BFT: Biofeedback Training; EMG: Electromyography; NIRS: Near-Infrared Spectroscopy; PET: Positron Emission Tomography; CW: Continuous Wave; MLB: Modified Lambert-Beer; GLM: General Linear Model

\section{Introduction}

The electromyogram (EMG) is frequently used to monitor motion and muscle performance during biofeedback (BF) training (BFT). Pioneered by Jacobson at Harvard University in 1908, the use of BF techniques resulted in the progressive muscle relaxation technique [1]. Indeed, progressive muscle relaxation is used to control anxiety and is thought to encourage self-care and enhance overall health [2]. Gatchel and Price, Gaarder and Montgomery and Basmajian et al. comprehensively reviews the applications and historical perspectives of $\mathrm{BF}$, which are beyond the scope of this discussion [3-5]. BFT is not only a technique used for enhancing health, but is predicted to affect the development and maintenance of brain function [6,7]. The connection between BFT and brain function surely will garner further attention in future, as current studies state that BFT involves not only voluntary movement of the extremities and joints, but receives input from high-level integrative functions of the brain.

Recently, it has been shown that hip flexor muscles, used to bend the hip joint when walking, rapidly reduce in mass with age. Hip joint flexors include the rectus femoris and abdominal muscles, which have been implicated as being related to falls in elderly people. BF techniques allow subjects to observe EMG signals or signal-derived outputs in order to encourage self-control of a specific muscle. This technique has been used to develop a technique for the "local exercise" of muscles including the rectus femoris, thus contributing to fall prevention and health enhancement in the elderly. Instruction is frequently provided using a visual or auditory signal.

One technique for non-invasive measurement of brain activity that has been developed in recent years is brain functional imaging using near-infrared spectroscopy (NIRS). With developments such as the miniaturization of diagnostic equipment, brain science is developing rapidly and a variety of brain activities are being defined. Additionally, NIRS requires the subject to be restrained to a lesser degree than with other techniques, such as functional magnetic resonance imaging (fMRI) and positron emission tomography (PET) [8-10].

NIRS measures changes in hemoglobin $(\mathrm{Hb})$ concentration in the blood. There is a limitation to the wavelength range at which near infrared light is absorbed in the body [11], however, near infrared light scattered into brain tissue from above the scalp, can reach the cerebral 
cortex [12]. The cerebral cortex is located at a depth of 15-20 mm from the scalp and displays a close correlation between neural activity and capillary constriction or dilation. The cerebral cortex is therefore suitable for measuring changes in intracerebral hemoglobin concentration associated with brain activity. Moreover, the cerebral cortex is also critically linked to movement, sensation, language and cognition. During NIRS of the cerebral cortex, activity is measured using multichannel reflection measurements from the scalp. Briefly, light-emitting probes and light-receiving probes are placed on the scalp and then near infrared light (wavelength of 700-900 nm), with high permeability into biotissue, is emitted from these light-emitting probes. The light-receiving probes then detect light that is scattered and reflected by the cerebral cortex.

Blood contains two types of hemoglobin, namely oxygenated hemoglobin $(\mathrm{Oxy}-\mathrm{Hb})$ that is bound to oxygen and deoxygenated hemoglobin (Deoxy-Hb) that is not bound to oxygen and the absorption spectra differs for both [11]. The present study uses this characteristic to measure Oxy-Hb concentration (Co) and Deoxy-Hb concentration $(\mathrm{Cd})$ using the continuous wave $(\mathrm{CW})$ method, based on the attenuation of detected light versus the intensity of the near infrared light reflection at two wavelengths, $\lambda 1$ and $\lambda 2$. The CW method is based on the modified Lambert-Beer (MLB) method and measures the concentration change from the start of recording multiplied by the optical path $[11,13]$. This method is useful as the human body scatters light strongly, meaning the direct optical path from emission to detection cannot be reliably measured and therefore, obtained values do not represent absolute Hb levels.

Measurements using NIRS assume neurovascular coupling in the same way as other measuring techniques [13]. Neurovascular coupling in the brain refers to blood vessels dilating near active nerves, in order that arterial blood that contains high levels of oxygen and glucose can be supplied, with associated changes in Oxy-Hb and Deoxy-Hb [14]. Additionally, active states in brain regions can be estimated by measuring changes in cerebral blood flow. In fact, it has been demonstrated that localized $\mathrm{Hb}$ increase and decrease $(\mathrm{Co}, \mathrm{Cd})$ reflect cerebral activity $[15,16]$.

In brain activity measurements using NIRS, the prefrontal cortex (related to working memory, control of attention, cognition and emotion) and the premotor cortex (related to planning and preparing for motion) are activated during low-load "full-body movement" (e.g. walking) [17]. In fact, studies report that cognitive function in elderly people is improved through walking exercise [18]. Furthermore, previous work by the authors demonstrated the possibility that specific "local movement" (masticatory movement in this case) stimulates activity of the prefrontal cortex [19].

In the present study, measurements were taken from the rectus femoris using EMG and NIRS simultaneously for healthy young subjects, whose physiological properties are not markedly different from those of healthy elderly subjects, to reveal the effect of BFT on the brain and in particular the prefrontal cortex. In addition, the effect of BFT exercise tasks on local cerebral blood flow was investigated.

\section{Methodology}

\section{Participants}

Biometric data were obtained for the rectus femoris muscle in seven healthy young individuals ( $22 \pm 1$ year) with no abnormalities in the extremities and no past medical history of ear or nervous system disease.

\section{Materials}

Surface EMG tests were performed by connecting an EMG transformation box (AP-U027, TEAC Co., Tokyo) to a commercially available portable multi-purpose biosignal amplifier-embedded collection device (Polymate AP1532, TEAC Co., Tokyo) and by using dedicated bipolar EMG electrodes with pre-amps. Furthermore, an AP Monitor (NoruPro, Tokyo) was used to obtain PC recordings at a 2 $\mathrm{kHz}$ sampling frequency to show muscle activity to the subjects in real time. Subjects were given full explanations prior to undergoing the tests and consent was obtained in writing. The ethics committee of the department of human and artificial intelligent systems, graduate school of engineering, university of Fukui, approved all experiments used in this study.

\section{Procedure}

The following outline summarizes the experimental process:

Step 1: Subjects were asked to sit back on a chair (with four fixed legs) and to kick with their dominant leg against a belt, attached to the lower part of the chair (Figure 1).

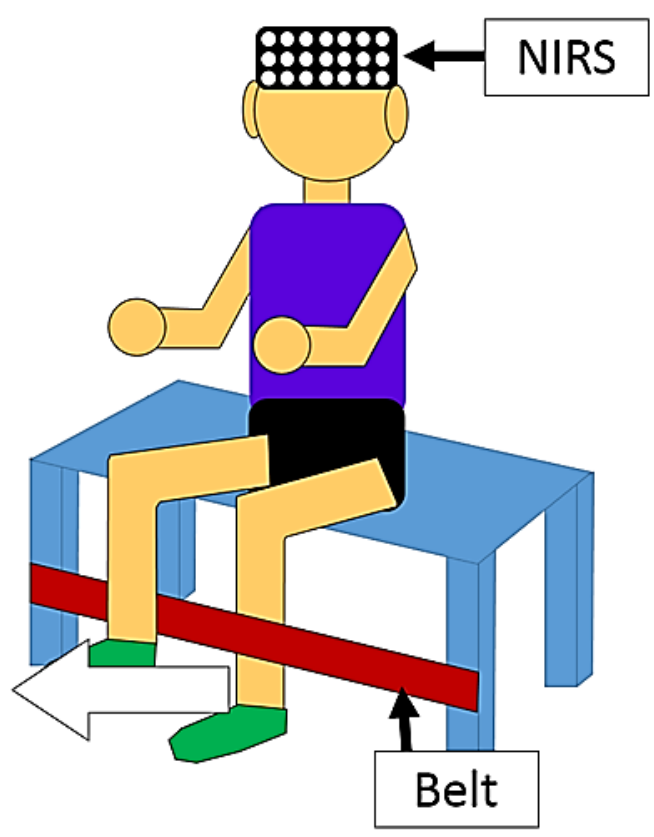

Figure 1: Biofeedback training experiment for rectus femoris muscle.

Step 2: Away from the center of the rectus femoris, AMG electrodes were placed at intervals of a few centimeters and subjects were asked to perform their maximum voluntary contraction. The average integral waveform of the surface EMG was calculated for this period of muscle contraction and muscular activity corresponding to $\alpha=75 \%$ (third quartile) of maximum voluntary contraction was then estimated. 
Step 3: Muscular activity corresponding to $\alpha \%$ of maximum voluntary contraction was shown to the subject as the instruction signal and five cycles of intermittent signals were provided for 40 seconds of contraction (gradual build-up during the first 20 seconds), hereafter referred to as the "transient period", followed by 20 seconds of constant muscle activity, hereafter referred to as the "muscle contraction period") and then 40 seconds of relaxation (the first 20 seconds are referred to as the pre-rest and the last 20 seconds as postrest) (Figure 2).

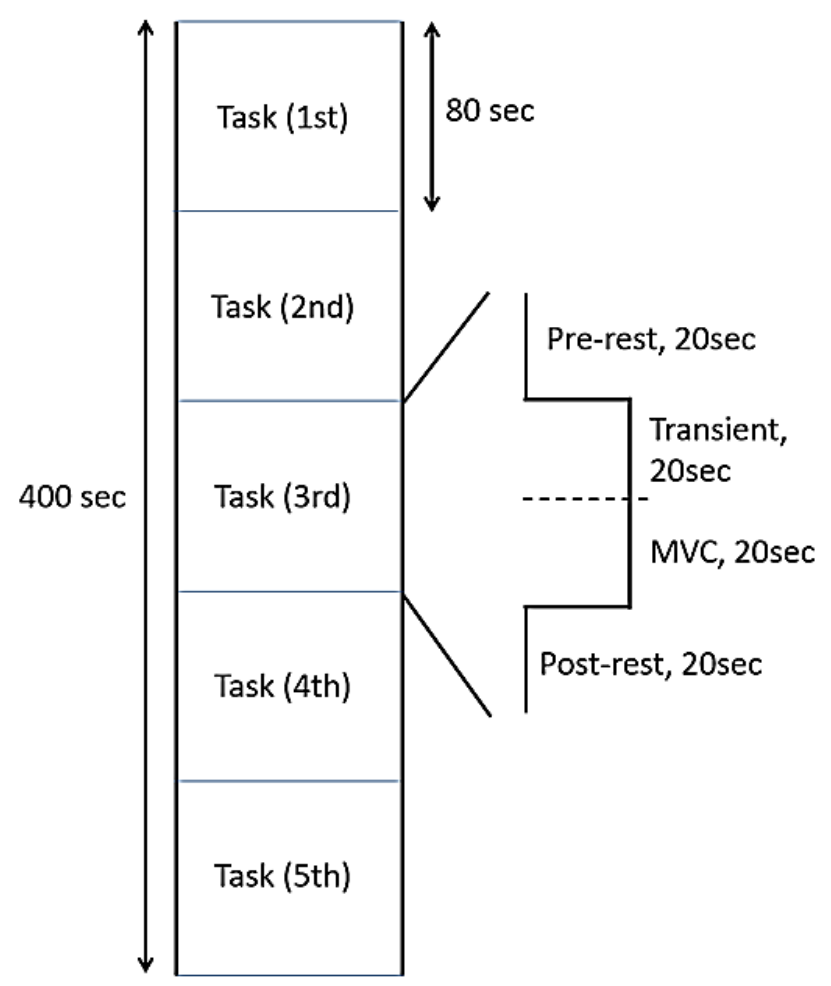

Figure 2: Experiment protocol.

The EMG waveforms obtained over 400 seconds were rectified and smoothed in real time at 0.1 second intervals of integration and these integral waveforms were then shown to subjects (in addition to the instruction signals). Cut-off frequency for the high and low range cutoff filters was set at $1 \mathrm{kHz}$ and $16 \mathrm{kHz}$, noise was removed from the surface EMG by inserting an $\mathrm{AC}$ removal filter and evaluation performed through a "sensor output signal evaluation system" [20].

Step 4: In conjunction with Step 3, the optical brain function imaging device LABNIRS (Shimadzu Corporation, Kyoto) was used to measure $\mathrm{Co}$ and $\mathrm{Cd}$ at a sampling frequency of $7.7 \mathrm{~Hz}[13,21]$. A holder was placed on the subject's head, with light-emitting/receiving probes arranged based on international 10-20 sensor placement as shown in Figure 3. Changes in cerebral blood flow concentrations were measured on the frontal lobe, at 54 channels (Figure 3). Owing to space limitations, this manuscript focuses on the analysis of Co only.

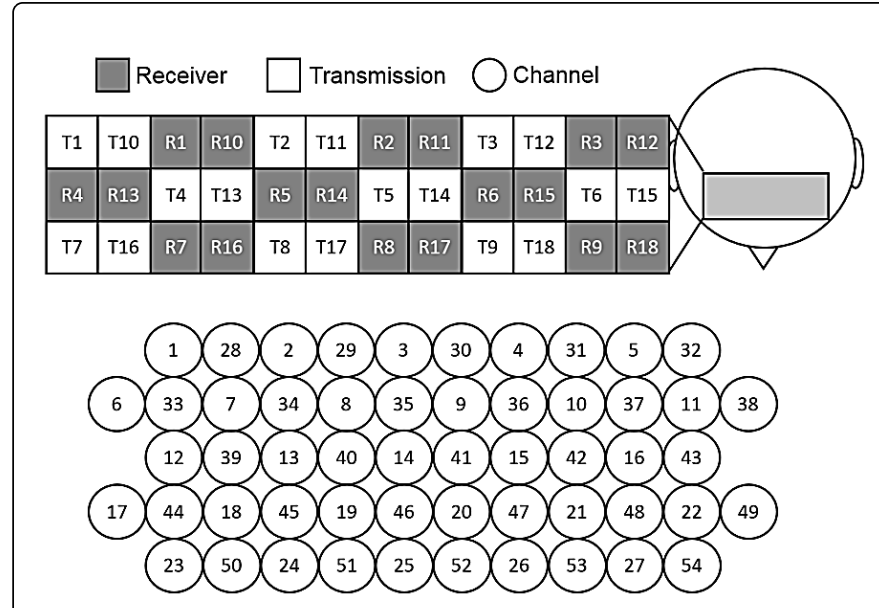

Figure 3: Array of irradiation and light-receiving probes.

\section{Data analysis}

For each subject, standardization was performed using average Co in the pre-rest period in each BFT cycle and the standard deviation. After taking the arithmetic mean of the pre-rest, transient, muscle contraction and post-rest periods for every sampling time, average Co was calculated across all subjects. For each pre-rest, transient, muscle contraction and post-rest period we calculated the average, $\mu$, standard deviation, $\sigma$, skewness and kurtosis of their distribution for Co for each channel and we also confirmed the form of the distribution itself. Whether the distribution for each period can be regarded as normal distribution $N\left(\mu, \sigma^{2}\right)$ was verified using a chi-squared test and whether a difference could be deemed to exist in the population mean for Co in the pre-rest period and Co in the muscle contraction period, was checked using a Welch's $t$ test. The significance level was set at 0.05 .

\section{Results}

We recorded time series data of local cerebral blood flow on the frontal lobe, at 54 channels (Figure 4). BFT results were checked with a sensor output signal evaluation system. Muscle performance follow-up of the instruction signals was good for all subjects.

For each pre-rest, transient, muscle contraction and post-rest period we calculated the average, standard deviation, skewness and kurtosis of distribution for Co for each channel. Co, which was broadly 0 in the pre-rest period, increased to a few $[\mathrm{mMcm}]$ for every measured location (ch) through the transient period and in the muscle contraction period. The Welch's $t$ test revealed that Co in the muscle contraction period was also significantly increased for all channels compared to Co in the pre-rest period. The degree of freedom for $t$ distribution was 643 and the t boundary value (two-sided test) for level of significance 0.05 was 1.96 .

Next, we confirmed the shape of the distribution relating to cerebral blood flow changes for each pre-rest, transient, muscle contraction and post-rest period. Figure 4 shows a typical example. Distribution is close to normal in the pre-rest period, during BFT the distribution is flattened and multiple peaks are seen occasionally (Figure 4). 


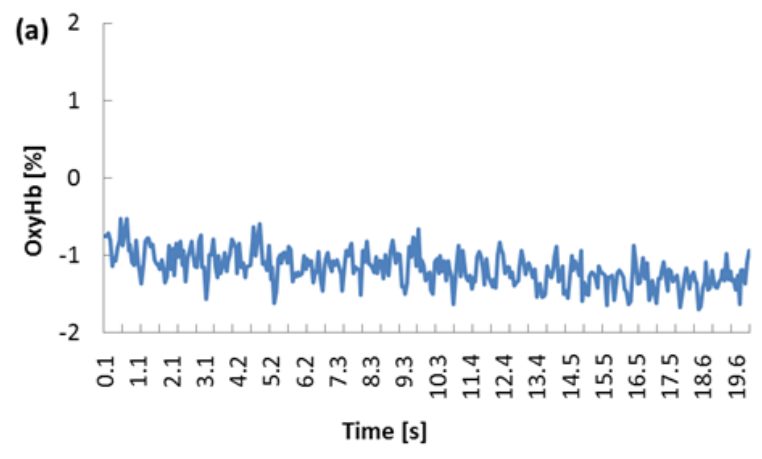

(b) 2

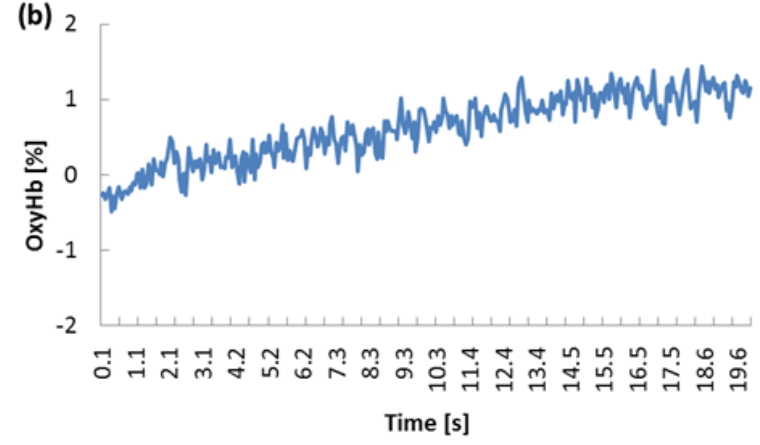

Figure 4: Typical example of time series data of Oxy-Hb on ch-18; (a) at pre-rest; (b) during muscle contraction.

Using the average, $\mu$ and standard deviation, $\sigma$, for each period recorded we asked whether Co distribution for each period could be considered as a normal distribution $\mathrm{N}\left(\mu, \sigma^{2}\right)$, the findings were statistically corroborated using a chi-squared test. However, in all locations normal distribution could not be observed during the muscle contraction period.

\section{Discussion}

We investigated the effect of BFT exercise tasks on changes in local cerebral blood flow in the prefrontal cortex. In all channels, Co during a muscle contraction period was significantly higher than in the prerest period $(\mathrm{p}<0.05)$. We propose that the kicking action has the potential to alter brain function.

Regarding skewness and kurtosis of Co distribution, although it was close to 0 in the pre-rest period and demonstrates characteristics of normal distribution, these characteristics were not observed on the non-parietal side of the prefrontal cortex. During BFT, almost all ch recorded were negative values and no characteristics of normal distribution were observed. In fact, a flattened distribution and multiple peaks were seen occasionally during the muscle contraction period (Figure $4 \mathrm{~b}$ ). In addition, we asked whether Co distribution for each period could be considered as a normal distribution $\mathrm{N}\left(\mu, \sigma^{2}\right)$, with the findings being statistically corroborated using a chi-squared test. When the degree of freedom is set to 9 and standard deviation is set to 0.05 , the chi-squared boundary value is 19.02 . At the nonparietal side of the prefrontal cortex almost none of the ch could be regarded as having normal distribution, even in the pre-rest period (Figure 5).
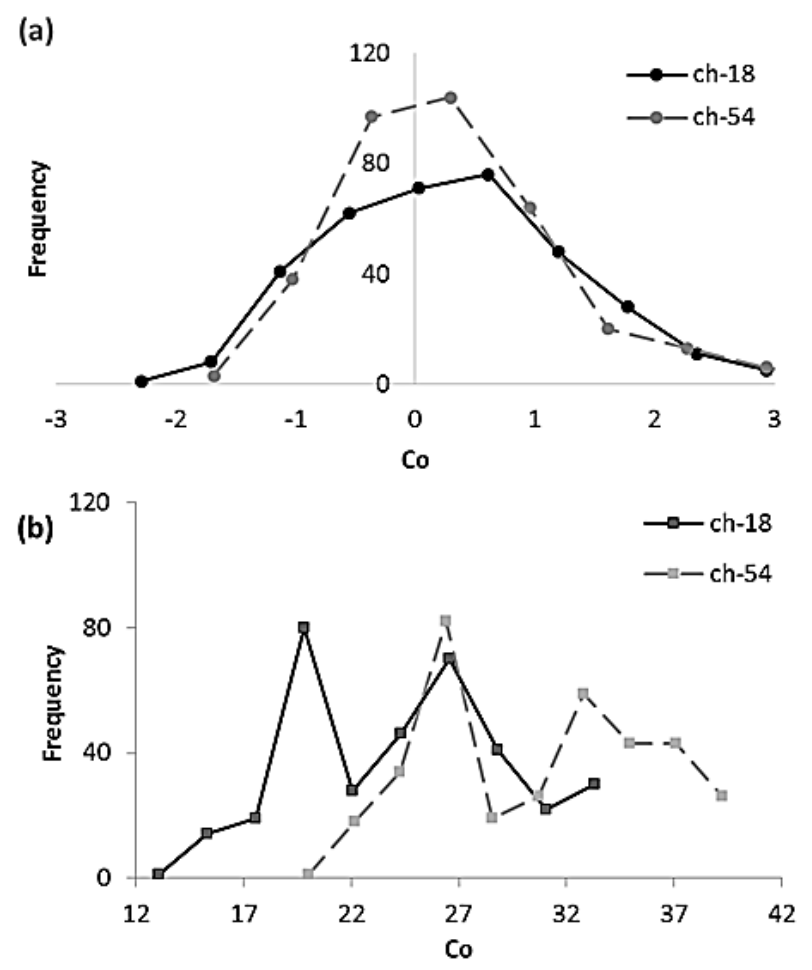

Figure 5: Typical example of distribution of Co on ch-18, 54; (a) at pre-rest; (b) during muscle contraction.

As mentioned previously, measurements through NIRS assume neurovascular coupling. The following assumptions are additionally made, due to the restrictions of the mathematical model (linear matrix) used by the CW method [11].

First assumption: $\mathrm{Hb}$ changes only occur in the brain. The agents causing near infrared light absorption changes are only $\mathrm{Oxy}-\mathrm{Hb}$ and Deoxy-Hb in the brain.

Second assumption: The optical path length factor existing in the coefficient matrix is not dependent on wavelength.

Third assumption: The constant term in the MLB method (degree of light reduction due to scattering) is regarded as constant during measuring.

Specifically, as a result of first assumption, tasks resulting in changes in skin sympathetic nerve activity associated with exposure to heat and cold or tasks resulting in sudden peripheral heat dissipation associated with excessive exercise, are not measured using the CW method or NIRS. Full-body movement not only brings about a physiological response on a peripheral level, but it is also possible that it acts on the endogenous pain control system through the prefrontal cortex and the hypothalamus, due to activity of the motor and the premotor cortices. This is of significant interest [22], but can generally not be verified through measuring brain function. On the other hand, limited exercise such as BFT is believed to be suitable for measuring through NIRS.

In principle, measurements of locations where optical path length is believed to differ cannot be compared. However, an analytical method that is independent of optical path length is known where signals are 
Page 5 of 6

regarded as instances of probability events and statistics are measured and this can be applied irrespective of the presence of any activation [23]. A general linear model (GLM) was used for the statistical processing, where we assumed that signals at the time of brain activation are expressed as a linear sum of a number of elementary functions and errors. As has been revealed here, based on the times series relating to changes in cerebral blood flow when performing exercise tasks, distributions were observed that deviate significantly from normal distribution and we anticipate the complexity of these generators will require careful analysis.

In general, there is no consensus on the characteristics of the lowpass filter needed for evaluating cerebral blood flow dynamics. Moreover, no mathematical model has been established that describe cerebral blood flow dynamics.

In this study, we examine whether nonlinear stochastic differential equations are appropriate as mathematical models to describe cerebral blood flow dynamics. With the change in cerebral blood flow during BFT as the target, we estimated the translation error to quantify the degree of determinism in the mathematical models describing it. When estimating the translation error from the time-series data by gradually lowering the cut-off frequency in the low-pass filter, the value decreased (Figure 6).

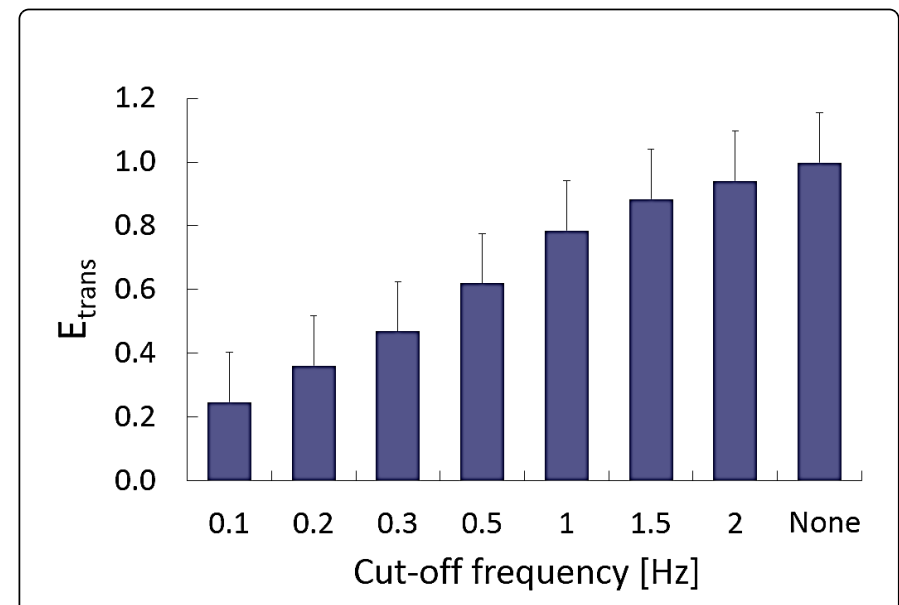

Figure 6: Variation of translation error with cut-off frequency.

If we set the threshold value for determinism to 0.5 , mathematical models describing the change in cerebral blood flow become qualitatively different, with cut-off frequency of $0.3-0.5 \mathrm{~Hz}$ as the boundary and can be perceived as different mathematical models. In other words, if we set the cut-off frequency below this range, artifacts are removed from the time-series data, but there is also a possibility that the characteristics of the original time-series data could be greatly altered.

Similarly, we checked the nonlinearity of the mathematical models of the time-series data using the surrogate data method by gradually lowering the cut-off frequency in the low-pass filter (Figure 7).

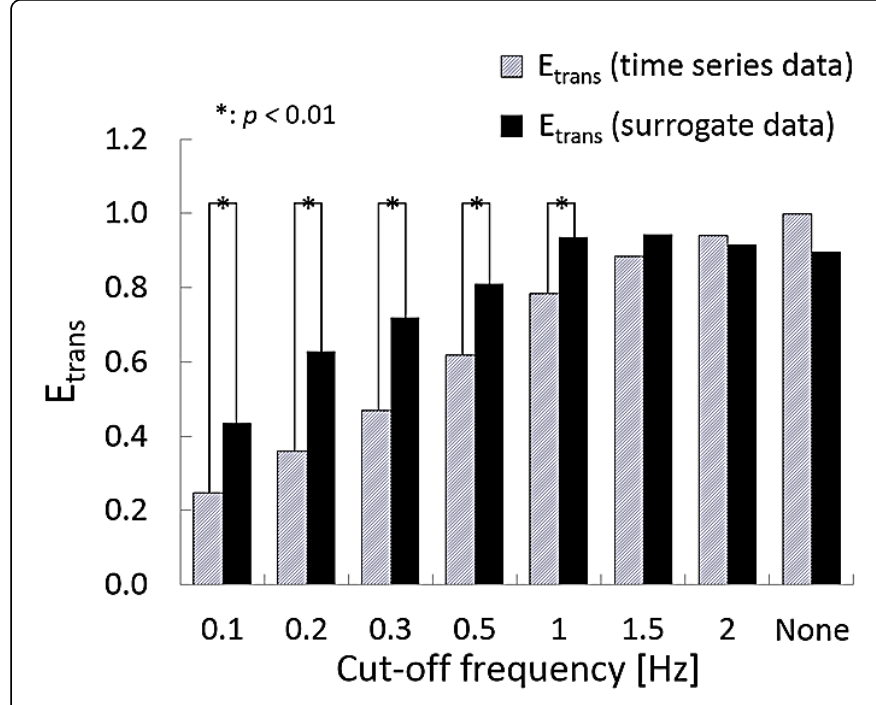

Figure 7: Results of nonlinearity in time-series data using surrogate data method and Wayland algorithm with cut-off frequency.

With the cut-off frequency set below $1 \mathrm{~Hz}$, we were able to capture the nonlinearity in mathematical models describing the time series. These results suggest the possibility that by setting the cut-off frequency of the low-pass filter to be $0.2 \mathrm{~Hz}$ or above but below $1 \mathrm{~Hz}$, mathematical models for cerebral blood flow dynamics can be appropriately captured and they can be captured as nonlinear stochastic differential equations.

There is a possibility that enhancement of the working memory by the proposed method can contribute actively to the treatment, also prevention of the related disease with previous studies on clinical medicine $[24,25]$.

In future, we plan to conduct a coherence analysis for surface EMGs measured under step 3 and a time series relating to changes in Oxy$\mathrm{Hb}$, Deoxy-Hb and Total-Hb concentration measured under step 4.

\section{Conclusion}

We investigated the effect of BFT exercise tasks on changes in local cerebral blood flow in the prefrontal cortex. Changes in Oxy-Hb concentration in the muscle contraction period were found to be increased compared to the values in the pre-rest period. This suggests that the kicking action alters brain function and in particular, that it may activate working memory. Furthermore, based on the times series relating to changes in cerebral blood flow while performing exercise tasks, a distribution was observed that deviates significantly from the normal distribution. In future, we intend to discuss the complexity of these generators from multiple angles and generate a mathematical model.

\section{Acknowledgement}

This work was supported in part by Descente and Ishimoto Memorial Foundation for the Promotion of Sports Science and Kayamori Foundation of Informational Science Advancement. 
Citation: Matsuura Y, Tanimura T, lida D, Takada H (2018) Local Cerebral Blood Flow during Biofeedback Training. J Sports Med Doping Stud 8: 205. doi:10.4172/2161-0673.1000205

Page 6 of 6

\section{Conflict of Interest}

None declared.

\section{References}

1. Jacobson E (1938) Progressive Relaxation. University of Chicago Press, Chicago, USA.

2. Snyder M, Lindquist R (1998) Complementary Alternative Therapies in Nursing. 3rd edn. Springer Publishing, New York, USA.

3. Gatchel RJ, Price KP (1979) Critical Applications of Biofeedback. Pergamon Press, New York, USA.

4. Gaarder KR, Montgomery PS (1981) Clinical Biofeedback: A Procedura Manual for Behavioral Medicine. Williams \& Wilkins, Baltimore, USA.

5. Basmajian S (1989) An Anthology of Visual Poetry and Collage. Sober Minute Press, Chicago, USA.

6. Calomeni MR, Rocha JA, Silva AP, Ribeiro LH, Marques L, et al. (2013) Brain stimulation used as biofeedback training for recovery of motor functions deteriorated by stroke. Arq Neuropsiquiatr 71: 159-164

7. Nanhoe-Mahabier W, Allum JH, Pasman EP, Overeem S, Bloem BR (2012) The effects of vibrotactile biofeedback training on trunk sway in Parkinson's disease patients. Parkinsonism Relat Disord 18: 1017-1021.

8. Villringer A, Dirnagl U (1995) Coupling of brain activity and cerebral blood flow: Basis of functional neuroimaging. Cerebrovasc Brain Metab Rev 7: 240-276.

9. Villringer A (1997) Non-invasive optical spectroscopy and imaging of human brain function. Trends Neurosci 20: 435-442.

10. Okamoto M, Dan H, Sakamoto K, Takeo K, Shimizu K, et al. (2004) Three-dimensional probabilistic anatomical cranio-cerebral correlation via the international 10-20 system oriented for transcranial functional brain mapping. NeuroImage 21: 99-111.

11. Shimura T, Amida T (2009) Prefrontal Lobe Measurement Using Near Infrared Spectroscopy: Evaluation of Early Detection Methods and Rehabilitation Methods of Dementia. Corona publishing, Tokyo, Japan.

12. Watanabe E, Murota Y, Nakajima C (2005) Functional mapping of language activities during the recovery phase of aphasia using near infrared optical topography. High Brain Funct Res 25: 215-223.
13. Zardecki A (1983) Multiple scattering corrections to the Beer-Lambert law. Proc SPIE 410: 103-111.

14. Komozumi H (1997) Observe the active brain: Visualization of higher brain function. Chem Today 11:27-33.

15. Hoshi Y, Tamura M (1993) Detection of dynamic changes in cerebral oxgenation coupled to neural function during mental work in man. Neurosci Lett 150: 5-8.

16. Kato T, Kamei A, Takashima S, Ozaki T (1993) Human visual cortical function during photic stimulation monitoring by means of near-infared spectroscopy. J Cereb Blood Flow Metab 13: 516-520.

17. Miyai I, Tanabe H, Sase I, Eda H, Oda I, et al. (2001) Cortical mapping of gait in human: A near-infrared spectroscopic topography study. NeuroImage 14: 1186-1192.

18. Middleton LE, Mitnitski A, Fallah N, Kirkland SA, Rockwood K (2008) Changes in cognition and mortality in relation to exercise in late life: A population based study. PLoS One 3: e3124.

19. Matsuura Y, Taniguchi T, Sugiura A, Miyao M, Takada H (2012) Distribution of cerebral blood flow during gum-chewing. Forma 27: 1-4.

20. Shiozawa T, Takada H, Miyao M (2006) Sensor output signal evaluation system. Japan Patent P2006-111387.

21. Kato T, Kamei A, Takashima S, Ozaki T (1993) Human visual cortical function during photic stimulation monitoring by means of near-infrared spectroscopy. J Cereb Blood Flow Metab 13: 516-520.

22. Taylor AF, Kuo FE (2008) Children with attention deficits concentrate better after walk in the park. J Atten Disord 23: 36-56.

23. Friston KJ, Holmes AP, Worsley KJ, Poline JP, Frith CD, et al. (1995) Statistical parametric maps in functional imaging: A general linear approach. Hum Brain Mapp 2: 189-210.

24. Colcombe S, Kramer AF (2003) Fitness effects on the cognitive function of older adults: A meta-analytic study. Psychol Sci 14: 125-130.

25. Harada T, Miyai I, Suzuki M, Kubota K (2009) Gait capacity affects cortical activation patterns related to speed control in the elderly. Exp Brain Res 193: 445-454. 